\title{
A GEOMETRIC CONSTRUCTION OF TYPES FOR THE SMOOTH REPRESENTATIONS OF PGL(2) OVER A LOCAL FIELD
}

\author{
PAUL BROUSSOUS
}

Dedicated to Guy Henniart on his 60th birthday

\begin{abstract}
We show that almost all (Bushnell and Kutzko) types of PGL(2, F), $F$ a non-Archimedean locally compact field of odd residue characteristic, naturally appear in the cohomology of finite graphs.
\end{abstract}

\section{INTRODUCTION}

Let $F$ be a non-Archimedean locally compact field and $G$ the group PGL $(2, F)$. We assume that the residue characteristic of $F$ is not 2 . In previous works ([2], [3]) we defined a tower of directed graphs $\left(\tilde{X}_{n}\right)_{n \geq 0}$ lying $G$-equivariantly over the Bruhat-Tits tree $X$ of $G$. We proved the two following facts:

Theorem 1 ([3], Theorem (3.2.4), page 502). Let $(\pi, \mathcal{V})$ be a non-spherical generic smooth irreducible representation. Then $(\pi, \mathcal{V})$ is a quotient of the cohomology space with compact support $H_{c}^{1}\left(\tilde{X}_{n(\pi)}, \mathbb{C}\right)$, where $n(\pi)$ is the conductor of $\pi$.

Theorem 2 ([3], Theorem (5.3.2), page 512). If $(\pi, \mathcal{V})$ is a supercuspidal smooth irreducible representation of $G$, then we have

$$
\operatorname{dim}_{\mathbb{C}} \operatorname{Hom}_{G}\left[H_{c}^{1}\left(\tilde{X}_{n(\pi)}, \mathbb{C}\right), \mathcal{V}\right]=1 .
$$

In this paper we make the $G$-module structure of $H_{c}^{1}\left(\tilde{X}_{n}, \mathbb{C}\right)$ more explicit for all $n \geq 0$, and draw some interesting consequences.

Let us fix an edge $\left[s_{0}, s_{1}\right]$ of $X$ and denote by $\mathcal{K}_{0}$ and $\mathcal{K}_{1}$ the stabilizers in $G$ of $s_{0}$ and $\left[s_{0}, s_{1}\right]$, respectively. Then $\mathcal{K}_{0}$ and $\mathcal{K}_{1}$ form a set of representatives of the two conjugacy classes of maximal compact subgroups in $G$. If $n$ is even, we have a $G$-equivariant mapping $p_{n}: \tilde{X}_{n} \longrightarrow X$ which respects the graph structures. We denote by $\Sigma_{n}$ the subgraph $p_{n}^{-1}\left(\left[s_{0}, s_{1}\right]\right)$. If $n$ is odd, then after passing to the first barycentric subdivisions, we have a $G$-equivariant mapping $p_{n}: \tilde{X}_{n} \longrightarrow X$ which respects the graph structures. We denote by $\Sigma_{n}$ the subgraph $p_{n}^{-1}\left(S\left(s_{0}, 1 / 2\right)\right)$, where $S\left(s_{0}, 1 / 2\right)$ denotes the set of points $x$ in $X$ such that $d\left(x, s_{0}\right) \leq 1 / 2$ (here $d$ is the natural distance on the standard geometric realization of $X$, normalized in such a way that $\left.d\left(s_{0}, s_{1}\right)=1\right)$.

Then for all $n, \Sigma_{n}$ is a finite graph, equipped with a an action of $\mathcal{K}_{1}$ if $n$ is even, and $\mathcal{K}_{0}$ if $n$ is odd. So the cohomology spaces $H^{1}\left(\Sigma_{n}, \mathbb{C}\right)$ provide finite dimensional smooth representations of $\mathcal{K}_{1}$ or $\mathcal{K}_{0}$, according to the parity of $n$.

Received by the editors March 26, 2012 and, in revised form, April 17, 2013.

2010 Mathematics Subject Classification. Primary 22E50; Secondary 20J05.

The author wants to thanks the anonymous referee whose remarks helped him to improve the presentation of this article. 
For each $n \geq 0$, we define an finite set $\mathcal{P}_{n}$ of pairs $(\mathcal{K}, \lambda)$ formed of a maximal compact subgroup $\mathcal{K} \in\left\{\mathcal{K}_{0}, \mathcal{K}_{1}\right\}$ and of an irreducible smooth representation of $\mathcal{K}$. By definition we have $(\mathcal{K}, \lambda) \in \mathcal{P}_{n}$ if and only if there exists $k \in\{0,1, \ldots, n\}$ such that $(\mathcal{K}, \lambda)$ is an irreducible constituent of the representation $H^{1}\left(\Sigma_{k}, \mathbb{C}\right)$. For $(\mathcal{K}, \lambda) \in \mathcal{P}_{n}$ and $k \leq n$, we denote by $m_{\lambda}^{k}$ the multiplicity of $\lambda$ in $H_{c}^{1}\left(\Sigma_{k}, \mathbb{C}\right)$ and we set $m_{n, \lambda}=m_{\lambda}=m_{\lambda}^{0}+\cdots+m_{\lambda}^{n}$. Note that $m_{\lambda}$ depends on $(\mathcal{K}, \lambda)$ and $n$.

The main results of this article are the following.

Theorem A. For all $n \geq 0$, we have the direct sum decomposition

$$
H_{c}^{1}\left(\tilde{X}_{n}, \mathbb{C}\right)=\mathbf{S t}_{G} \oplus \bigoplus_{(\mathcal{K}, \lambda) \in \mathcal{P}_{n}}\left(c-\operatorname{ind}_{\mathcal{K}}^{G} \lambda\right)^{m_{\lambda}}
$$

(Here $\mathbf{S t}_{G}$ denotes the Steinberg representation of $G$ ).

Theorem B. For all $n \geq 0$, any element of $\mathcal{P}_{n}$ is:

a) either a type in the sense of Bushnell and Kutzko's type theory [6], which is not a type for the unramified principal series,

b) or a pair of the form $\left(\mathcal{K}_{0}, \chi \circ \operatorname{det} \otimes \mathbf{S t}_{\mathcal{K}_{0}}\right)$, where $\chi$ is a smooth character of $F^{\times}$of order 2 , trivial on the group of 1-units in $F^{\times}$, and $\mathbf{S t}_{\mathcal{K}_{0}}$ is the representation inflated from the Steinberg representation of $\mathrm{PGL}(2)$ of the residue field of $F$,

c) or the pair $\left(\mathcal{K}_{1}, \mathbf{1}_{\mathcal{K}_{1}}\right)$, where $\mathbf{1}$ denotes a trivial character.

It is worth noting that if the pairs of cases a) and b) are not types, they are typical in the sense of 9 .

Corollary C. Let $n \geq 0$. If $(\mathcal{K}, \lambda) \in \mathcal{P}_{n}$ is a cuspidal type, then $m_{n, \lambda}=1$.

Indeed this follows from Theorems 2 and A using Frobenius reciprocity for compact induction.

By Theorem 1, any Bernstein component of $G$, different from the unramified principal series component, must have a type in $\mathcal{P}_{n}$ for $n$ large enough. Hence the graphs $\tilde{X}_{n}, n \geq 0$, provide a geometric construction of types for almost all Bernstein components of $G$.

We conjecture that if $(\mathcal{K}, \lambda) \in \mathcal{P}_{n}$ is a type of $G$, then $m_{\lambda}=1$.

Finally, let us observe that this construction gives a new proof that the irreducible supercuspidal representations of $G$ are obtained by compact induction. Our proof differs from Kutzko's original proof ([10, also see [4]) only at the exhaustion steps. Indeed our "supercuspidal" types are the same as Kutzko's, but we prove that any irreducible supercuspidal representation contains such a type by using an argument based on [2] and [3], that is mainly on the existence of the new vector.

The article is organized as follows. The proof of Theorem A relies first on combinatorial properties of the graphs $\tilde{X}_{n}$ that are stated and proved in $\S 2$. Using these combinatorial properties and some homological arguments, we show in $\S 3$ how to relate the cohomology of $\tilde{X}_{n}$ to that of $\tilde{X}_{n-1}$. The irreducible components of $H^{1}\left(\Sigma_{n}\right)$ are determined in $\S 4$ when $n$ is even, and in $\S 5$ and $\S 6$ when $n$ is odd. A synthesis of the arguments of paragraphs 2 to 6 , leading to a proof of Theorems A and $\mathrm{B}$, is given in $\S 7$.

We shall assume that the reader is familiar with the language of Bushnell and Kutzko's type theory [6] and with the language of strata ([5], 4]). 


\section{NotATion}

We shall denote by

$F$ a non-Archimedean non-discrete locally compact field,

$\mathfrak{o}$ its valuation ring,

$\mathfrak{p}$ the maximal ideal of $\mathfrak{o}$,

$\varpi$ the choice of a uniformizer of $\mathfrak{o}$,

$\mathbf{k}=\mathfrak{o} / \mathfrak{p}$ the residue field of $F$,

$p$ the characteristic of $\mathbf{k}$,

$q=p^{f}$ the cardinal of $\mathbf{k}$,

$G$ the group $\operatorname{PGL}(2, F)$.

$t_{\varpi}$ the image of the matrix $\left(\begin{array}{ll}1 & 0 \\ 0 & \varpi\end{array}\right)$ in $G$.

The results of this article are obtained by the following.

Hypothesis. The characteristic of $\mathbf{k}$ is not 2

We shall often define an element, a subset, or a subgroup of $G$ by giving a (set of) representative(s) in $\mathrm{GL}(2, F)$.

We write $T$ for the diagonal torus of $G$ and $B \supset T$ for the upper standard Borel subgroup. We denote by $T^{0}$ the maximal compact subgroup of $T$, i.e., the set of matrices with coefficients in $\mathfrak{o}^{\times}$, and by $T^{n}$ the subgroup of matrices with coefficients in $1+\mathfrak{p}^{n}, n>0$.

Let $k, l$ be integers satisfying $k+l \geq 0$. Then

$$
\mathfrak{A}(k, l)=\left(\begin{array}{cc}
\mathfrak{o} & \mathfrak{p}^{l} \\
\mathfrak{p}^{k} & \mathfrak{o}
\end{array}\right)
$$

is an $\mathfrak{o}$-order of $\mathrm{M}(2, F)$. We denote by $\Gamma_{0}(k, l)$ the image in $G$ of its group of units. There are two conjugacy classes of maximal compact subgroups of $G$. The first one has representative $K=\Gamma_{0}(0,0)$. A representative $\tilde{I}$ of the second one is the semidirect product of the cyclic group generated by $\Pi=\left(\begin{array}{cc}0 & 1 \\ \varpi & 0\end{array}\right)$ with the Iwahori subgroup $I=\Gamma_{0}(1,0)$.

The group $K$ is filtered by the normal compact open subgroups

$$
K_{n}=\left(\begin{array}{cc}
1+\mathfrak{p}^{n} & \mathfrak{p}^{n} \\
\mathfrak{p}^{n} & 1+\mathfrak{p}^{n}
\end{array}\right), n \geq 1
$$

The group $I$ is filtered by the normal compact subgroups $I_{n}, n \geq 1$, defined by

$$
I_{2 k+2}=\left(\begin{array}{cc}
1+\mathfrak{p}^{k+1} & \mathfrak{p}^{k+1} \\
\mathfrak{p}^{k+2} & 1+\mathfrak{p}^{k+1}
\end{array}\right), I_{2 k+1}=\left(\begin{array}{cc}
1+\mathfrak{p}^{k+1} & \mathfrak{p}^{k} \\
\mathfrak{p}^{k+1} & 1+\mathfrak{p}^{k+1}
\end{array}\right), k \geq 0
$$

The subgroups $I_{n}, n \geq 1$, are normalized by $\Pi$.

We denote by $X$ the Bruhat-Tits building of $G$. This is a uniform tree with valency $q+1$. As a $G$-set and as a simplicial complex $X$ identifies with the following complex. Its vertices are the homothety classes $[L]$ of full $\mathfrak{o}$-lattices $L$ in the vector space $V=F^{2}$. Two vertices $[L]$ and $[M]$ define an edge if and only if there exists a basis $\left(e_{1}, e_{2}\right)$ of $V$ such that, up to homothety, we have $L=\mathfrak{o} e_{1} \oplus \mathfrak{o} e_{2}$ and $M=\mathfrak{o} e_{1} \oplus \mathfrak{p} e_{2}$.

The vertices of the standard apartment (i.e., the apartment stabilized by $T$ ) are the $s_{k}=\left[\mathfrak{o} \oplus \mathfrak{p}^{k}\right], k \in \mathbb{Z}$. The element $t_{\varpi}$ acts as $t_{\varpi} s_{k}=s_{k+1}, k \in \mathbb{Z}$. The maximal compact subgroup $K$ is the stabilizer of $s_{0}$ and $\tilde{I}$ (resp. $I$ ) is the global stabilizer (resp. pointwise stabilizer) of the edge $\left[s_{0}, s_{1}\right]$. If $l \geq k$, the pointwise stabilizer of the segment $\left[s_{k}, s_{l}\right]$ is $\Gamma_{0}(l,-k)$. 


\section{Combinatorics of $\tilde{X}_{n}$}

We recall the construction of the directed graphs $\tilde{X}_{n}, n \geq 1$.

For any integer $k \geq 1$, an oriented $k$-path in $X$ is an injective sequence of vertices $\left(s_{i}\right)_{i=0, \ldots, k}$ in $X$ such that, for $k=0, \ldots, k-1,\left\{s_{i}, s_{i+1}\right\}$ is an edge in $X$. We shall allow the index $i$ to run over any interval of integers of length $k+1$. Let us fix an integer $n \geq 1$. The directed graph $\tilde{X}_{n}$ is constructed as follows. Its edge set (resp. vertex set) is the set of oriented $(n+1)$-paths (resp. $n$-paths) in $X$. If $a=\left\{s_{0}, s_{1}, \ldots, s_{n+1}\right\}$ is an edge of $\tilde{X}_{n}$, its head (resp. its tail) is $a^{+}=$ $\left\{s_{1}, s_{2}, \ldots, s_{n+1}\right\}$ (resp. $a^{-}=\left\{s_{0}, s_{1}, \ldots, s_{n}\right\}$ ). The graphs we obtain in this way are actually simplicial complex. The group $G$ acts on $\tilde{X}_{n}$ is an obvious way; the action preserves the structure of directed graph.

When $n=2 m$ is even, we have a natural simplicial projection $p=p_{n}: \tilde{X}_{n} \rightarrow X$ given on vertices by $p\left(s_{-m}, \ldots, s_{0}, \ldots, s_{m}\right)=s_{0}$. It is $G$-equivariant. Let $e=$ $\left\{s_{0}, t_{0}\right\}$ be an edge of $X$. We are going to describe the finite simplicial complex $p^{-1}(e)$. An edge in $\tilde{X}_{n}$ above the edge $e$ corresponds to an oriented $(2 m+1)$-path of one of the following forms:

i) $\left(s_{-m}, s_{-m+1}, \ldots, s_{0}, t_{0}, \ldots, t_{m-1}, t_{m}\right)$,

ii) $\left(t_{-m}, t_{-m+1}, \ldots, t_{0}, s_{0}, \ldots, s_{m-1}, s_{m}\right)$.

Let $C_{2 m-1}(e)$ be the set of $(2 m-1)$-paths $c=\left(s_{-m+1}, \ldots, s_{0}, t_{0}, \ldots, t_{m-1}\right)$. We say that $c \in C_{2 m-1}(e)$ lies above e. Fix $c \in C_{2 m-1}(e)$ and consider the simplicial subcomplex $\tilde{X}_{2 m}[e, c]$ of $\tilde{X}_{2 m}$ whose edges correspond to the $(2 m+1)$-paths of the form

$$
\left(a, s_{-m+1}, \ldots, s_{0}, t_{0}, \ldots, t_{m-1}, b\right) .
$$

So $a$ (resp. $b$ ) can be any neighbour of $s_{-m+1}\left(\right.$ resp. $\left.t_{m-1}\right)$ different from $s_{-m+2}$ (resp. $t_{m-1}$ ), with the convention that $s_{1}=t_{0}$ and $t_{-1}=s_{0}$. The simplicial complex $\tilde{X}_{2 m}[e, c]$ is connected. It is indeed isomorphic to the complete bipartite graph with sets of vertices:

$\left\{a ; a\right.$ neighbour of $\left.s_{-m+1}, a \neq s_{-m+2}\right\}$ and $\left\{b ; b\right.$ neighbour of $\left.t_{m-1}, b \neq t_{m-2}\right\}$.

Lemma 3.1. Let $e$ and $e^{\prime}$ be two edges of $X$ and $c \in C_{2 m-1}(e), c^{\prime} \in C_{2 m-1}\left(e^{\prime}\right)$. Then $\tilde{X}_{2 m}[e, c] \cap \tilde{X}_{2 m}\left[e^{\prime}, c^{\prime}\right] \neq \emptyset$ if and only if we are in one of the following cases:

i) $e=e^{\prime}$ and $c=c^{\prime}$ (so that $\tilde{X}_{2 m}[e, c]=\tilde{X}_{2 m}\left[e^{\prime}, c^{\prime}\right]$ );

ii) $e \cap e^{\prime}$ is reduced to one vertex of $X$ and $c \cup c^{\prime}$ is an oriented $2 m$-path in $X$. In that case $\tilde{X}_{2 m}[e, c] \cap \tilde{X}_{2 m}\left[e^{\prime}, c^{\prime}\right]$ is reduced to the vertex of $\tilde{X}_{2 m}$ corresponding to the $2 m$-path $c \cup c^{\prime}$.

Proof. If $\tilde{X}_{2 m}[e, c] \cap \tilde{X}_{2 m}\left[e^{\prime}, c^{\prime}\right] \neq \emptyset$, then $e \cap e^{\prime}=p\left(\tilde{X}_{2 m}[e, c]\right) \cap p\left(\tilde{X}_{2 m}\left[e^{\prime}, c^{\prime}\right]\right) \neq \emptyset$. Assume first that $e=e^{\prime}$. Then $c=c^{\prime}$, for if $c \neq c^{\prime}$, then $\tilde{X}_{2 m}[e, c] \cap \tilde{X}_{2 m}\left[e^{\prime}, c^{\prime}\right]=\emptyset$; indeed, if $\tilde{s}$ is a vertex of $\tilde{X}_{2 m}[e, c]$, then it determines $c$ uniquely. Now assume that $e \cap e^{\prime}$ is a vertex. Let $\tilde{s} \in \tilde{X}_{2 m}[e, c] \cap \tilde{X}_{2 m}\left[e^{\prime}, c^{\prime}\right]$. Then $\tilde{s}$ contains $c$ and $c^{\prime}$ as subsequences, with $c \neq c^{\prime}$. So by a length argument $s=c \cup c^{\prime}$. Conversely if $c \cup c^{\prime}$ is an oriented $2 n$-path, then $c \cup c^{\prime}$ is a vertex of $\tilde{X}$ lying in $\tilde{X}_{2 m}[e, c] \cap \tilde{X}_{2 m}\left[e^{\prime}, c^{\prime}\right]$.

Corollary 3.2. For any edge e of $X$, the connected components of $p^{-1}(e)$ are the $\tilde{X}_{2 m}[e, c]$, where $c$ runs over $C_{2 m-1}(e)$.

Define a 1-dimensional simplicial complex $Y_{2 m-1}$ in the following way. Its vertices are the connected components $\tilde{X}_{2 m}[e, c]$, where $e$ runs over the edges of $X$ and $c$ 
over $C_{2 m-1}(e)$, and two vertices $\tilde{X}_{2 m}[e, c]$ and $\tilde{X}_{2 m}\left[e^{\prime}, c^{\prime}\right]$ are linked by an edge if they intersect. Note that $Y_{2 m-1}$ is naturally a $G$-simplicial complex.

Corollary 3.3. As a $G$-simplicial complex, $Y_{2 m-1}$ is canonically isomorphic to the complex $\tilde{X}_{2 m-1}$.

Lemma 3.4. For all $n \geq 0$, the simplicial complex $\tilde{X}_{n}$ is connected.

Proof. When $n$ is even this is [2, Lemma (4.1)]. For odd $n$, the proof is similar.

Assume that $m \geq 1$. We say that an edge of $\tilde{X}_{2 m-1}$ lies above a vertex $s_{0}$ of $X$ if as an oriented $2 m$-path it has the form $\left(s_{-m}, \ldots, s_{o}, \ldots, s_{m}\right)$. For any vertex $s_{0}$ of $X$ we write $\tilde{X}_{2 m-1}\left[s_{o}\right]$ for the subsimplicial complex of $\tilde{X}_{2 m-1}$ formed of the edges lying above $s_{0}$.

Lemma 3.5. When $m=1$ the simplicial complexes $\tilde{X}_{2 m-1}\left[s_{0}\right]=\tilde{X}_{1}\left[s_{0}\right]$ are connected.

Proof. We may identify the neighbour vertices of $s_{0}$ in $X$ with the points of the projective line $\mathbb{P}^{1}(\bar{M}) \simeq \mathbb{P}^{1}(\mathbf{k})$, where $s_{0}=[M]$ and $\bar{M}=M / \mathfrak{p} M$. The vertices of $\tilde{X}_{1}\left[s_{0}\right]$ are the oriented 1-paths $\left(s_{0}, x\right),\left(y, s_{0}\right), x, y \in \mathbb{P}^{1}(\bar{M})$. Two oriented 1-paths of the form $\left(x, s_{0}\right)$ and $\left(s_{0}, y\right)$ are linked by the edge $\left(x, s_{0}, y\right)$. Let $\left(x, s_{0}\right),\left(y, s_{0}\right)$ be two oriented 1-paths with $x \neq y$. Since $\left|\mathbb{P}^{1}(\mathbf{k})\right| \geq 3$, there exists $z \in \mathbb{P}^{1}(\bar{M})$ distinct from $x$ and $y$. Then $\left(x, s_{0}\right)$ is linked to $\left(s_{o}, z\right)$ via the path $\left(x, s_{0}, z\right)$ and $\left(s_{0}, z\right)$ is linked to $\left(y, s_{0}\right)$ via the path $\left(y, s_{0}, z\right)$. For vertices of the form $\left(s_{0}, x\right),\left(s_{0}, y\right)$ the proof is similar.

We now assume that $m>1$. We write $C_{2 m-2}\left(s_{0}\right)$ for the set $(2 m-2)$-paths of the form $\left(s_{-m+1}, \ldots, s_{0}, \ldots, s_{m-1}\right)$. For any $c \in C_{2 m-2}\left(s_{0}\right)$, we consider the subsimplicial complex $\tilde{X}_{2 n-1}\left[s_{0}, c\right]$ of $\tilde{X}_{2 m-1}$ whose edges corresponds to the $2 m$ paths of the form $\left(a, s_{-n+1}, \ldots, s_{0}, s_{n-1}, b\right)$. We have results similar to Lemma 3.1 and Corollaries 3.2 and 3.3 .

Lemma 3.6. i) For any vertex $s_{0}$ of $X$ and for $c \in C_{2 m-2}\left(s_{0}\right), \tilde{X}_{2 m-1}\left[s_{0}, c\right]$ is connected. It is indeed isomorphic to a complete bipartite graph constructed on two sets of $q=|\mathbf{k}|$ elements.

ii) Let $s$ and $s^{\prime}$ be vertices of $X, c \in C_{2 m-2}(s)$ and $c^{\prime} \in C_{2 m-2}\left(s^{\prime}\right)$. Then $\tilde{X}_{2 m-1}[s, c] \cap \tilde{X}_{2 m-1}\left[s^{\prime}, c^{\prime}\right] \neq \emptyset$ if and only if $s=s^{\prime}$ and $c=c^{\prime}$, or $\left\{s, s^{\prime}\right\}$ is an edge in $X$ and $c \cup c^{\prime}$ is an oriented $2 n-1$-path. In this last case $\tilde{X}_{2 m-1}[s, c] \cap \tilde{X}_{2 m-1}\left[s^{\prime}, c^{\prime}\right]=$ $\{\tilde{s}\}$, where the vertex $\tilde{s}$ of $\tilde{X}_{2 m-1}$ corresponds to the $(2 n-1)$-path $c \cup c^{\prime}$.

iii) For any vertex $s$ of $X$, the connected components of $\tilde{X}_{2 m-1}[s]$ are $\tilde{X}_{2 m-1}[s, c]$, c running over $C_{2 m-2}(s)$.

We can consider the 1-dimensional simplicial complex $Z_{2 m-2}$ whose vertices are the connected components $\tilde{X}_{2 m-1}[s, c], s$ running over the vertices of $X$ and $c$ over $C_{2 m-2}(s)$, and where two connected components define an edge if and only if they intersect. Note that $Z_{2 m-2}$ is naturally a $G$-simplicial complex.

Corollary 3.7. As a $G$-simplicial complex $Z_{2 m-2}$ is isomorphic to $X_{2 n-2}$.

\section{The COHomology of $\tilde{X}_{n}$ : First Reductions}

If $\Sigma$ is a locally finite 1-dimensional simplicial complex, we write $\Sigma^{0}$ (resp. $\left.\Sigma^{(1)}, \Sigma^{1}\right)$ for its set of vertices (resp. non-oriented edges, oriented edges). We 
let $C_{0}(\Sigma)$ (resp. $C_{1}(\Sigma)$ ) denote the $\mathbb{C}$-vector space with basis $\Sigma^{0}$ (resp. $\Sigma^{1}$ ). We define the space $C_{c}^{0}(\Sigma, \mathbb{C})=C_{c}^{0}(\Sigma)\left(\right.$ resp. $\left.C_{c}^{1}(\Sigma, \mathbb{C})=C_{c}^{1}(\Sigma)\right)$ of oriented simplicial 0-cochains (resp. 1-cochains) with compact support by:

$C_{c}^{0}(\Sigma)=$ space of all linear forms $f: C_{0}(\Sigma) \rightarrow \mathbb{C}$ such that $f(s)=0$ except for a finite number of vertices $s$;

$C_{c}^{1}(\Sigma)=$ space of all linear forms $\omega: C_{1}(\Sigma) \rightarrow \mathbb{C}$ such that $\omega([a, b])=0$ except for a finite number of oriented edges $[a, b]$ and $\omega([a, b])=-\omega([b, a])$.

We set $C_{c}^{k}(\Sigma)=0$ for $k \in \mathbb{Z} \backslash\{0,1\}$ and define a coboundary map $d: C_{c}^{0}(\Sigma) \rightarrow$ $C_{c}^{1}(\Sigma)$ by $d f([a, b])=f(b)-f(a)$. The cohomology of the cochain complex $\left(C_{c}^{\bullet}(\Sigma), d\right)$ computes the cohomology with compact support $H_{c}^{\bullet}(\Sigma, \mathbb{C})=H_{c}^{\bullet}(\Sigma)$ of (the standard geometric realization of) $\Sigma$. If $\Sigma$ is acted upon by a group $H$ whose action is simplicial, then $\left(C_{c}^{\bullet}(\Sigma), d\right)$ is in a straightforward way a complex of $H$-modules, and its cohomology computes $H_{c}^{1}(\Sigma)$ as a $H$-module. When $\Sigma$ is finite we drop the subscripts $c$.

Since the stabilizer of a finite number of vertices of $X$ is open in $G$, we see that for $n \geq 1$, the $G$-modules $C_{c}^{0}\left(\tilde{X}_{n}\right), C_{c}^{1}\left(\tilde{X}_{n}\right)$ and therefore $H_{c}^{1}\left(\tilde{X}_{n}\right)$ are smooth.

In the sequel we fix $m \geq 1$ and we abbreviate $\tilde{X}_{2 m}=\tilde{X}$. The disjoint union $\tilde{X}^{1}=\bigsqcup_{e \in X^{(1)}} \tilde{X}_{e}$, where $\tilde{X}_{e}=p^{-1}(e)$, induces an isomorphism:

$$
\begin{array}{cc}
C_{c}^{1}(\tilde{X}) & \simeq \bigoplus_{e \in X^{(1)}} C^{1}\left(\tilde{X}_{e}\right), \\
\omega & \mapsto\left(\omega_{\mid C_{1}\left(\tilde{X}_{e}\right)}\right)_{e \in X^{(1)}} .
\end{array}
$$

Similarly, the non-disjoint union $\tilde{X}^{0}=\bigcup_{e \in X^{(1)}} \tilde{X}_{e}^{0}$ induces an injection:

$$
\begin{aligned}
j: C_{c}^{0}(\tilde{X}) & \hookrightarrow \bigoplus_{e \in X^{(1)}} C^{0}\left(\tilde{X}_{e}\right), \\
f & \mapsto\left(f_{\mid C_{0}\left(\tilde{X}_{e}\right)}\right)_{e \in X^{(1)}} .
\end{aligned}
$$

We have the following commutative diagram of $G$-modules:

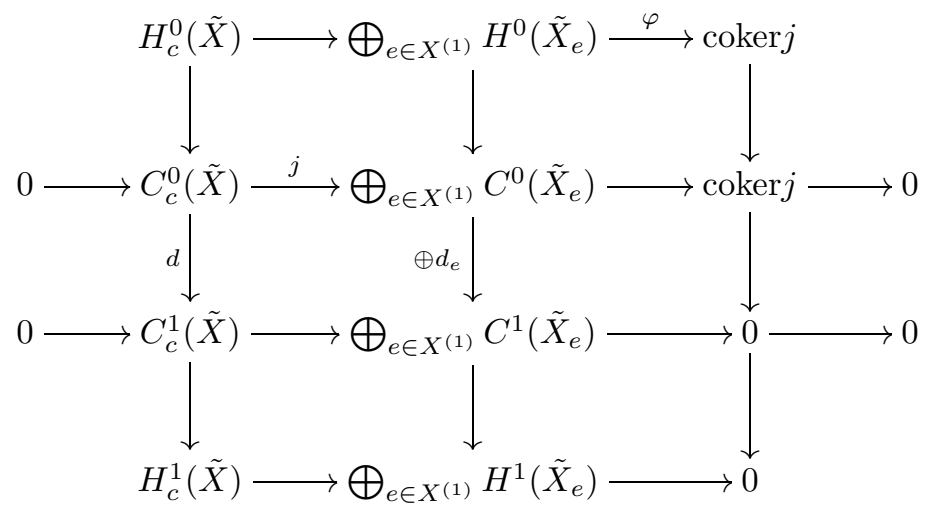

Here, for $e \in X^{(1)}, d_{e}$ denote the coboundary map $C^{0}\left(\tilde{X}_{e}\right) \rightarrow C^{1}\left(\tilde{X}_{e}\right)$. Since $\tilde{X}$ is connected ([2] Lemma 4.1) and non-compact, we have $H_{c}^{0}(\tilde{X})=0$. So the snake lemma gives the kernel-cokernel exact sequence:

$$
0 \rightarrow \bigoplus_{e \in X^{(1)}} H^{0}\left(\tilde{X}_{e}\right) \rightarrow \operatorname{coker} j \rightarrow H_{c}^{1}(\tilde{X}) \rightarrow \bigoplus_{e \in X^{(1)}} H^{1}\left(\tilde{X}_{e}\right) \rightarrow 0
$$

that is,

$$
0 \rightarrow \operatorname{cokerj} / \varphi\left(\bigoplus_{e \in X^{(1)}} H^{0}\left(\tilde{X}_{e}\right)\right) \rightarrow H_{c}^{1}(\tilde{X}) \rightarrow \bigoplus_{e \in X^{(1)}} H^{1}\left(\tilde{X}_{e}\right) \rightarrow 0 .
$$


Abbreviate $Y=Y_{2 m-1}$.

Lemma 4.1. We have a canonical isomorphism of $G$-modules

$$
\text { cokerj/ } \varphi\left(\bigoplus_{e \in X^{(1)}} H^{0}\left(\tilde{X}_{e}\right)\right) \simeq H_{c}^{1}(Y)
$$

Proof. From Corollary 3.2 we have

$$
\bigoplus_{e \in X^{(1)}} C^{0}\left(\tilde{X}_{e}\right)=\bigoplus_{e \in X^{(1)}} \bigoplus_{c \in C_{2 m-1}(e)} C^{0}\left(\tilde{X}_{2 m}[e, c]\right)
$$

So the map $j$ is given by $f \mapsto \bigoplus_{e \in X^{(1)}} \bigoplus_{c \in C_{2 m-1}(e)} f_{e, c}$, where $f_{e, c}=f_{\mid C_{0}\left(\tilde{X}_{2 m}[e, c]\right)}$. Consider the $G$-equivariant morphism of vector spaces

$$
\psi: \bigoplus_{e \in X^{(1)}} \bigoplus_{c \in C_{2 m-1}(e)} C^{0}\left(\tilde{X}_{2 m}[e, c]\right) \rightarrow C_{c}^{1}(Y)
$$

given as follows. If $\sigma$ is an oriented edge of $Y$, then their exist uniquely determined edges $e_{o}, e_{o}^{\prime}$ of $X, c_{o} \in C_{2 m-1}\left(e_{o}\right), c_{o}^{\prime} \in C_{2 m-1}\left(e_{o}^{\prime}\right)$, such that $\sigma$ corresponds to the intersection $\tilde{X}_{2 m}\left[e_{o}, c_{o}\right] \cap \tilde{X}_{2 m}\left[e_{o}^{\prime}, c_{o}^{\prime}\right]=\left\{s_{o}\right\}, s_{o} \in \tilde{X}^{0}$. We then set

$$
\psi\left[\left(f_{e, c}\right)_{e, c}\right](\sigma)=f_{e_{o}^{\prime}, c_{o}^{\prime}}\left(s_{o}\right)-f_{e_{o}, c_{o}}\left(s_{o}\right) .
$$

Then $\psi$ is surjective and its kernel is precisely $j\left(C_{c}^{0}(\tilde{X})\right)$. So we may identify coker $j$ with $C_{c}^{1}(Y)$. From Corollary 3.2, we have

$$
\bigoplus_{e \in X^{(1)}} H^{0}\left(\tilde{X}_{e}\right)=\bigoplus_{e \in X^{(1)}} \bigoplus_{c \in C_{2 m 1}(e)} H^{0}\left(\tilde{X}_{2 m}[e, c]\right)
$$

so that we may identify $\bigoplus_{e \in X^{(1)}} H^{0}\left(\tilde{X}_{e}\right)$ with $C_{c}^{0}(\tilde{Y})$. Under our identifications the map $\varphi: \bigoplus_{e \in X^{(1)}} H^{0}\left(\tilde{X}_{e}\right) \rightarrow$ coker $j$ corresponds to the coboundary map $d$ : $C_{c}^{0}(Y) \rightarrow C_{c}^{1}(Y)$, and we are done since all our identifications are $G$-equivariant.

Proposition 4.2. For $m \geq 1$, we have an isomorphism of $G$-modules:

$$
H_{c}^{1}\left(\tilde{X}_{2 m}\right) \simeq H_{c}^{1}\left(\tilde{X}_{2 m-1}\right) \oplus c-\operatorname{ind}_{\mathcal{K}_{e_{o}}}^{G} H^{1}\left(\tilde{X}_{e_{o}}\right)
$$

for any edge $e_{o}$ of $X$ and where $\mathcal{K}_{e_{o}}$ denotes the stabilizer of $e_{o}$ in $G$.

Proof. From the short exact sequence (4.3) and Lemma 4.1 we have the exact sequence of $G$-modules:

$$
0 \rightarrow H_{c}^{1}(Y) \rightarrow H_{c}^{1}(\tilde{X}) \rightarrow \bigoplus_{e \in X^{(1)}} H^{1}\left(\tilde{X}_{e}\right) \rightarrow 0 .
$$

Since $G$ acts transitively on the edges of $X, \bigoplus_{e \in X^{(1)}} H^{1}\left(\tilde{X}_{e}\right)$ identifies with the compactly induced representation $c$-ind $\mathcal{K}_{e_{o}}^{G} H^{1}\left(\tilde{X}_{e_{o}}\right)$. Moreover, this induced representation is projective in the category of smooth complex representations of $G$. This is classical and follows from Frobenius reciprocity for compact induction together with the fact that the category of smooth $\mathcal{K}_{e_{o}}$-modules is semisimple. So the sequence (4.4) splits.

We assume that $m \geq 1$ and we abbreviate $\tilde{X}=\tilde{X}_{2 m-1}$. The disjoint union $\tilde{X}^{1}=\bigsqcup_{s \in X^{0}} \tilde{X}_{2 m-1}[s]^{1}$ induces an isomorphism:

$$
\begin{aligned}
& C_{c}^{1}(\tilde{X}) \simeq \bigoplus_{s \in X^{0}} C^{1}\left(\tilde{X}_{2 m-1}[s]\right), \\
& \omega \quad \mapsto \quad\left(\omega_{\mid C_{1}\left(\tilde{X}_{2 m-1}[s]\right)}\right)_{s \in X^{0}} .
\end{aligned}
$$


Similarly, the non-disjoint union $\tilde{X}^{0}=\bigcup_{s \in X^{0}} \tilde{X}_{s}^{0}$ induces an injection:

$$
\begin{aligned}
j: C_{c}^{0}(\tilde{X}) & \hookrightarrow \bigoplus_{s \in X^{0}} C^{0}\left(\tilde{X}_{2 m-1}[s]\right), \\
f & \mapsto\left(f_{\mid C_{0}\left(\tilde{X}_{2 m-1}[s]\right)}\right)_{s \in X^{0}} .
\end{aligned}
$$

We have the following commutative diagram of $G$-modules:

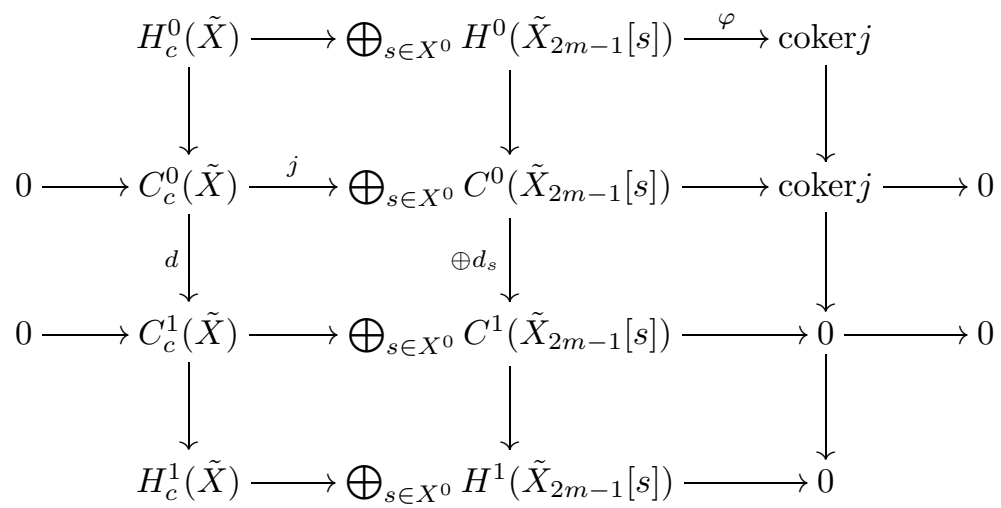

Here, for $s \in X^{0}, d_{s}$ denote the coboundary map $C^{0}\left(\tilde{X}_{2 m-1}[s]\right) \rightarrow C^{1}\left(\tilde{X}_{2 m-1}[s]\right)$. By Lemma 3.4, $\tilde{X}$ is connected. So we have $H_{c}^{0}(\tilde{X})=0$ since $\tilde{X}$ is non-compact. The snake lemma gives the kernel-cokernel exact sequence:

$$
0 \rightarrow \operatorname{coker} j / \varphi\left(\bigoplus_{s \in X^{0}} H^{0}\left(\tilde{X}_{2 m-1}[s]\right)\right) \rightarrow H_{c}^{1}(\tilde{X}) \rightarrow \bigoplus_{s \in X^{0}} H^{1}\left(\tilde{X}_{2 m-1}[s]\right) \rightarrow 0 .
$$

Lemma 4.3. We have a canonical isomorphism of $G$-modules

$$
\operatorname{coker} j / \varphi\left(\bigoplus_{s \in X^{0}} H^{0}\left(\tilde{X}_{2 m-1}[s]\right)\right) \simeq H_{c}^{1}\left(\tilde{X}_{2 m-2}\right) \text {. }
$$

Proof. It is similar to the proof of Lemma 4.1 and relies on Lemma 3.6 and Corollary 3.7.

Proposition 4.4. For $m \geq 1$, we have an isomorphism of $G$-modules:

$$
H_{c}^{1}\left(\tilde{X}_{2 m-1}\right) \simeq H_{c}^{1}\left(\tilde{X}_{2 m-2}\right) \oplus c \text {-ind }_{\mathfrak{K}_{s_{o}}}^{G} H^{1}\left(\tilde{X}_{s_{o}}\right)
$$

for any vertex $s_{o}$ and where $\mathcal{K}_{s_{o}}$ denotes the stabilizer of $s_{o}$ in $G$.

Proof. Similar to the proof of Proposition 4.2 .

Recall 3 that $\tilde{X}_{0}$ is different from $X$. This is a directed graph whose set of vertices is isomorphic to $X^{0}$ as a $G$-set and whose set of edges is isomorphic to the $G$-set of oriented edges of $X$.

\section{Determination of the inducing Representations - I}

Let $m \geq 0$ be a fixed integer and $e_{0}=\left[s_{0}, s_{1}\right]$ the standard edge. The aim of this section is to determine the $\mathcal{K}_{e_{0}}$-module $H^{1}\left(\tilde{X}_{2 m}\left[e_{0}\right]\right)$. Here we have $\mathcal{K}_{e_{0}}=\tilde{I}$, the normalizer in $G$ of the standard Iwahori subgroup. We have the semidirect products

$$
\tilde{I}=\left\langle\left(\begin{array}{cc}
0 & 1 \\
\varpi & 0
\end{array}\right)\right\rangle \ltimes I=E^{\times} I
$$


for any totally ramified subfield extension $E / F \subset \mathrm{M}(2, F)$ such that $E^{\times}$normalizes I.

We first assume that $m \geq 1$. By Corollary 3.2 , we have the disjoint union

$$
\tilde{X}_{2 m}\left[e_{0}\right]=\coprod_{c \in C_{2 m-1}\left(e_{0}\right)} \tilde{X}_{2 m}\left[e_{0}, c\right] \text {. }
$$

The group $\tilde{I}$ acts transitively on $C_{2 m-1}\left(e_{0}\right)$. This comes form the standard fact that $I$, the pointwise stabilizer of $e_{0}$ acts transitively on the apartments of $X$ containing $e_{0}$.

Let $c_{0} \in C_{2 m-1}\left(e_{0}\right)$ be the path

$$
s_{-m+1}, \ldots, s_{0}, s_{1}, \ldots, s_{m} .
$$

The global stabilizer of $\tilde{X}_{2 m}\left[e_{0}, c_{0}\right]$ in $\tilde{I}$ is the pointwise stabilizer of $c_{0}$ in $\tilde{I}$, that is,

$$
\Gamma_{0}(m, m-1)=\left(\begin{array}{cc}
\mathfrak{o}^{\times} & \mathfrak{p}^{m-1} \\
\mathfrak{p}^{m} & \mathfrak{o} \times
\end{array}\right)=T^{0} I_{2 m-1} .
$$

It follows that

$$
H^{1}\left(\tilde{X}_{2 m}\left[e_{0}\right]\right)=\operatorname{ind}_{T^{0} I_{2 m-1}}^{\tilde{I}} H^{1}\left(\tilde{X}_{2 m}\left[e_{0}, c_{0}\right]\right) .
$$

On the other hand, an easy calculation shows that the pointwise stabilizer of $\tilde{X}_{2 m}\left[e_{0}, c_{0}\right]$ is $T^{1} I_{2 m}$, where $T^{1}$ is the congruence subgroup of $T$ given by

$$
T^{1}=\left(\begin{array}{cc}
1+\mathfrak{p} & 0 \\
0 & 1+\mathfrak{p}
\end{array}\right) .
$$

So the $T^{0} I_{2 m-1}$-module $H^{1}\left(\tilde{X}_{2 m}\left[e_{0}, c_{0}\right]\right)$ may be viewed as a representation of the finite group $T^{0} I_{2 m-1} / T^{1} I_{2 m}$, that is, a semidirect product of the cyclic group $\mathbf{k}^{\times}$ with the abelian group $I_{2 m-1} / I_{2 m} \simeq \mathbf{k} \oplus \mathbf{k}$.

Set $\Gamma=\tilde{X}_{2 m}\left[e_{0}, c_{0}\right]$. This is a finite directed graph. Let $\Sigma_{-m}$ (resp. $\left.\Sigma_{m+1}\right)$ denote the set of vertices of $X$ that are neighbours of $s_{-m+1}$ and different from $s_{-m+2}$ (resp. neighbours of $s_{m}$ and different from $s_{m-1}$ ). Then the vertex set of $\Gamma$ is

$$
\begin{aligned}
\Gamma^{0} & =\left\{\left(a, s_{-m+1}, \ldots, s_{0}, \ldots, s_{m}\right) ; a \in \Sigma_{-m}\right\} \coprod\left\{\left(s_{-m+1}, \ldots, s_{0}, \ldots, s_{m}, b\right) ; b \in \Sigma_{m+1}\right\} \\
& \simeq \Sigma_{-m} \coprod \Sigma_{m+1}
\end{aligned}
$$

and its edge set is

$$
\Gamma^{1}=\left\{\left(a, s_{-m+1}, \ldots, s_{0}, \ldots, s_{m}, b\right) ; a \in \Sigma_{-m}, b \in \Sigma_{m+1}\right\} \simeq \Sigma_{-m} \times \Sigma_{m+1} .
$$

In particular, $\Gamma$ is a bipartite graph based on two sets of $q$ elements. In particular, its Euler character is given by

$$
\chi(\Gamma)=1-\operatorname{dim}_{\mathbb{C}} H^{1}(\Gamma)=2 q-q^{2},
$$

so that

$$
\operatorname{dim}_{\mathbb{C}} H^{1}(\Gamma)=q^{2}-2 q+1=(q-1)^{2} .
$$

Let $\mathbb{C}\left[\Gamma^{1}\right]$ be the space of complex function on $\Gamma^{1}$ and let $\mathcal{H}(\Gamma)$ be the space of harmonic 1-cochains on $\Gamma$ :

$$
\mathcal{H}(\Gamma)=\left\{f \in \mathbb{C}\left[\Gamma^{1}\right] ; \sum_{a \in \Gamma^{1}, s \in a}[a: s] f(a)=0, \text { all } s \in \Gamma^{0}\right\} .
$$


Here $[a: s]$ denote an incidence number. In our case:

$$
f \in \mathcal{H}(\Gamma) \text { iff }\left\{\begin{array}{l}
\sum_{a \in \Sigma_{-m}} f\left(a, s_{-m+1}, \ldots, s_{m}, b\right)=0, \quad \text { for all } b, \\
\sum_{b \in \Sigma_{m+1}} f\left(a, s_{-m+1}, \ldots, s_{m}, b\right)=0, \quad \text { for all } a .
\end{array}\right.
$$

This is a standard result (see e.g. [3, Lemma (1.3.2)]) that, as a $T^{0} I_{2 m-1} / T^{1} I_{2 m^{-}}$ module, $H^{1}(\Gamma)$ is isomorphic to the contragredient module of $\mathcal{H}(\Gamma)$.

An easy computation shows that we may identify $\Gamma^{1}$ with $\mathbf{k} \times \mathbf{k}$ in such a way that:

1) an element of $I_{2 m-1}=\left(\begin{array}{cc}1+\mathfrak{p}^{m} & \mathfrak{p}^{m-1} \\ \mathfrak{p}^{m} & 1+\mathfrak{p}^{m}\end{array}\right)$ acts as

$$
\left(1+\left(\begin{array}{cc}
\varpi^{m} a & \varpi^{m-1} b \\
\varpi^{m} c & \varpi^{m} d
\end{array}\right)\right) \cdot(x, y)=(x+\bar{b}, y+\bar{c})
$$

for $a, b, c, d \in \mathfrak{o}, x, y \in \mathbf{k}$, and

2) an element of $T^{0}$ acts as

$$
\left(\begin{array}{ll}
a & 0 \\
0 & d
\end{array}\right) \cdot(x, y)=\left(\bar{a} \bar{d}^{-1} x, \bar{d} \bar{a}^{-1} y\right)
$$

and the condition (5.3) gives us:

$$
f \in \mathcal{H}(\Gamma) \text { iff }\left\{\begin{array}{l}
\sum_{x \in \mathbf{k}} f(x, y)=0, \quad \text { for all } y \in \mathbf{k}, \\
\sum_{y \in \mathbf{k}} f(x, y)=0, \quad \text { for all } x \in \mathbf{k} .
\end{array}\right.
$$

A basis of $\mathbb{C}[\Gamma]$ is formed of the functions $\chi_{1} \otimes \chi_{2}(x, y)=\chi_{1}(x) \chi_{2}(y)$, where, for $i=1,2, \chi_{i}$ runs over the characters of $(\mathbf{k},+)$. It is clear that the $(q-1)^{2}$-dimensional subspace of $\mathbb{C}[\Gamma]$ generated by the $\chi_{1} \otimes \chi_{2}, \chi_{1} \not \equiv 1, \chi_{2} \not \equiv 1$, is contained in $\mathcal{H}(\Gamma)$. So using (5.2), we obtain:

$$
\mathcal{H}(\Gamma)=\operatorname{Span}\left\{\chi_{1} \otimes \chi_{2} ; \chi_{i} \in \widehat{\mathbf{k}}, \chi_{i} \not \equiv 1, i=1,2\right\} .
$$

It follows from (5.4) that, as an $I_{2 m-1} / I_{2 m}$-module, the space $\mathcal{H}(\Gamma)$ is the direct sum of 1-dimensional representations corresponding to the characters $\alpha=$ $\alpha\left(\chi_{1}, \chi_{2}\right), \chi_{i} \not \equiv 1, i=1,2$, given by

$$
\alpha\left(1+\left(\begin{array}{cc}
\varpi^{m} a & \varpi^{m-1} b \\
\varpi^{m} c & \varpi^{m} d
\end{array}\right)\right)=\chi_{1}(b) \chi_{2}(c) .
$$

In particular, $\mathcal{H}(\Gamma)$ is isomorphic to its contragredient and therefore isomorphic to $H^{1}(\Gamma)$ as an $I_{2 m-1} / I_{2 m}$-module. In the language of strata (the reader may refer to [4, $\S 4])$, for $\chi_{i} \not \equiv 1, i=1,2$, the character $\alpha\left(\chi_{1}, \chi_{2}\right)$ corresponds to a stratum of the form $[\mathcal{I}, 2 m, 2 m-1, \beta]$, where $\mathcal{I}$ is the standard Iwahori order and $\beta \in \mathrm{M}(2, F)$ is an element of the form $\Pi^{2 m-1}\left(\begin{array}{cc}u & 0 \\ 0 & v\end{array}\right), u, v \in \mathfrak{o}^{\times}$. In the terminology of [4, $\S 4$, page 98$]$ this stratum is a ramified simple stratum.

We now have enough material to prove the following result.

Proposition 5.1. Let $\lambda$ be an irreducible constituent of

$$
H^{1}\left(\tilde{X}_{2 m}\left[e_{0}\right]\right)=\operatorname{ind}_{T^{0} I_{2 m-1}}^{\tilde{I}} H^{1}\left(\tilde{X}_{2 m}\left[e_{0}, c_{0}\right]\right) .
$$


Then the compactly induced representation $c$-ind ${ }_{\tilde{I}}^{G} \lambda$ is irreducible, whence supercuspidal.

Proof. It is a standard result that an irreducible compactly induced representation is supercuspidal (see e.g. [8, page 194]).

The proof of the irreducibility is also standard by an argument due to Kutzko. But we repeat it for convenience. By Frobenius reciprocity, the restriction of $\lambda$ to $I_{2 m-1}$ contains a character $\alpha\left(\chi_{1}, \chi_{2}\right)$ corresponding to a (ramified) simple stratum. Since $\lambda$ is irreducible and since $\tilde{I}$ normalizes $I_{2 m-1}$, the restriction $\lambda_{\mid I_{2 m-1}}$ is a direct sum $\alpha_{1} \oplus \cdots \oplus \alpha_{r}$ of $\tilde{I}$-conjugates of $\alpha\left(\chi_{1}, \chi_{2}\right)$. They all correspond to simple strata. Let $g \in G$ be an element intertwining $\lambda$ with itself. Then by restriction it intertwines a character $\alpha_{i}$ with a character $\alpha_{j}$ for some $j=1, \ldots, r$. By [4, Lemma (16.1), page 111], such an element $G$ must belong to $\tilde{I}$. It follows that the $G$ intertwining of $\lambda$ is equal to $\tilde{I}$ and that the representation $c$-ind $\tilde{I}_{\tilde{I}}^{G} \lambda$ is irreducible according to Mackey's irreducibility criterion ([8, Proposition (1.5), page 195]).

We finally consider the case $m=0$. The directed graph $\tilde{X}_{0}$ has $X^{0}$ as vertex set. An edge $\{t, s\}$ in $X$ gives rise to two edges $[s, t]$ and $[t, s]$ in $\tilde{X}_{0}$. Since the action of $G$ on $\tilde{X}_{0}$ preserves the structure of the digraph, the $G$-module $H_{c}^{1}\left(\tilde{X}_{0}\right)$ may be computed using the complex

$$
0 \longrightarrow C_{c}^{0}\left(\tilde{X}_{0}\right) \longrightarrow C_{c}^{(1)}\left(\tilde{X}_{0}\right)
$$

where $C_{c}^{(1)}\left(\tilde{X}_{0}\right)$ is the space of (unoriented) 1-cochains, that is the space of maps from $\tilde{X}_{0}^{(1)}$ (unoriented edges) to $\mathbb{C}$ with finite support. The coboundary map is given here by $d f(a)=f\left(a^{+}\right)-f\left(a^{-}\right)$. Consider the $G$-equivariant injection $j$ : $C_{c}^{1}(X) \longrightarrow C_{c}^{(1)}\left(\tilde{X}_{0}\right)$ given by $j(\omega):[s, t] \mapsto \omega([s, t])$. We have the commutative diagram of $G$-modules:

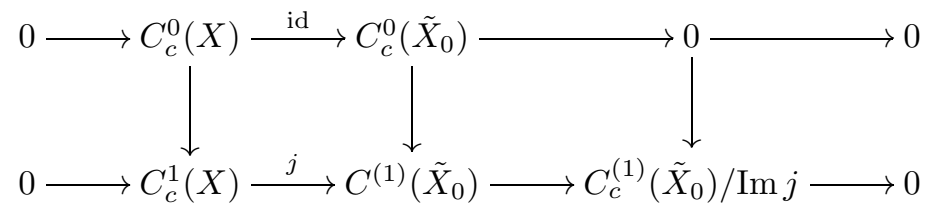

The quotient $C_{c}^{(1)}\left(\tilde{X}_{0}\right) / \operatorname{Im} j$ identifies with the subspace of $C_{c}^{(1)}\left(\tilde{X}_{0}\right)$ formed by those functions $f$ satisfying $f([s, t])=f([t, s])$ for all edges $\{s, t\}$ of $X$. This subspace is nothing more than the compactly induced representation $\mathrm{c}-\operatorname{Ind}_{\tilde{I}}^{G} \mathbf{1}_{\tilde{I}}$. The cokernel exact sequence gives us:

$$
0 \longrightarrow H_{c}^{1}(X) \longrightarrow H_{c}^{1}\left(\tilde{X}_{0}\right) \longrightarrow c-\operatorname{ind}_{\tilde{I}}^{G} \mathbf{1}_{\tilde{I}} \longrightarrow 0 .
$$

Now we use the following two facts to obtain Proposition 5.2:

- the representation $c$-ind $\tilde{I}_{\tilde{I}}^{G} \mathbf{1}_{\tilde{I}}$ is a projective object of the category of smooth representations of $G$,

- the $G$-module $H_{c}^{1}(X)$ is isomorphic to the Steinberg representation $\mathbf{S t}_{G}$ of $G$ ([7]).

Proposition 5.2. The G-module $H_{c}^{1}\left(\tilde{X}_{0}\right)$ is isomorphic to $\mathbf{S t}_{G} \oplus c$-ind ${ }_{\tilde{I}}^{G} \mathbf{1}_{\tilde{I}}$. 


\section{The inducing RePRESEntations - II}

We now determine the $\mathcal{K}_{s_{0}}$-module $H^{1}\left(\tilde{X}_{2 m+1}\left[s_{0}\right]\right)$. The arguments are very often similar to those of the previous section and we will not give all details. Since the case $m=0$ requires slightly different techniques we postpone it to the end of the section and assume first that $m>0$.

Recall that the stabilizer $\mathcal{K}_{s_{0}}$ of $s_{0}$ in $G$ is the image $K$ of $\mathrm{GL}(2, \mathfrak{o})$ in $G$.

Let $c_{0} \in C_{2 m}\left(s_{0}\right)$ be the path $\left(s_{-m}, \ldots, s_{0}, \ldots, s_{m}\right)$. Its pointwise stabilizer is $\Gamma_{0}(m, m)=T^{0} K_{m}$. So as a $K$-module, $H^{1}\left(\tilde{X}_{2 m+1}\left[s_{0}\right]\right)$ is isomorphic to the induced representation $\operatorname{Ind}_{T^{0} K_{m}}^{K} H^{1}\left(\tilde{X}_{2 m+1}\left[s_{0}, c_{0}\right]\right)$. Moreover, the pointwise stabilizer of $\tilde{X}_{2 m+1}\left[s_{0}, c_{0}\right]$ is $T^{1} K_{m+1}$ and $H^{1}\left(\tilde{X}_{2 m+1}\left[s_{0}, c_{0}\right]\right)$ may be viewed as a representation of $T^{0} K_{m} / T^{1} K_{m+1}$.

As in the previous section, one may consider the bipartite graph $\Omega$ whose both vertice sets identify with $\mathbf{k}$, equipped with an action of $K_{m}$ on $\Omega^{1}$ given by

$$
\left[I_{2}+\varpi^{m}\left(\begin{array}{cc}
a & b \\
c & d
\end{array}\right)\right] \cdot(x, y)=(x+\bar{b}, y+\bar{c}),
$$

the action of $T^{0}$ being given by

$$
\left(\begin{array}{ll}
a & 0 \\
0 & d
\end{array}\right) \cdot(x, y)=\left(\bar{a} \bar{d}^{-1} x, \bar{d} \bar{a}^{-1} y\right)
$$

Then the contragredient of the $T^{0} K_{m} / T^{1} K_{m+1}$-module $H^{1}\left(\tilde{X}_{2 m+1}\left[s_{0}, c_{0}\right]\right)$ is isomorphic to the space $\mathcal{H}(\Omega)$ of harmonic cochains on $\Omega$. As in the previous section the latter space is generated by the functions $\chi_{1} \otimes \chi_{2}$, where $\chi_{i}, i=1,2$, runs over the non-trivial characters of $(k,+)$. The line $\mathbb{C} \chi_{1} \otimes \chi_{2}$ is acted upon by $K_{m}$ via the character $\alpha\left(\chi_{1}, \chi_{2}\right)$ given by

$$
\alpha\left(\chi_{1}, \chi_{2}\right)\left(I_{2}+\varpi^{m}\left(\begin{array}{ll}
a & b \\
c & d
\end{array}\right)\right)=\chi_{1}(b) \chi_{2}(c) .
$$

It follows that $\mathcal{H}(\Omega)$ is isomorphic to its contragredient and that $H^{1}\left(\tilde{X}_{2 m+1}\left[s_{0}, c_{0}\right]\right)$ is the direct sum of the characters $\alpha\left(\chi_{1}, \chi_{2}\right), \chi_{i} \not \equiv 1, i=1,2$.

For $\chi_{i} \not \equiv 1, i=1,2$, the character $\alpha\left(\chi_{1}, \chi_{2}\right)$ corresponds to a stratum of the form $[\mathrm{M}(2, \mathfrak{o}), m, m-1, \beta]$, where $\beta \in \mathrm{M}(2, F)$ is given by $\varpi^{-m}\left(\begin{array}{ll}0 & u \\ v & 0\end{array}\right), u, v \in \mathfrak{o}^{\times}$. This stratum is either simple and non-scalar or split fundamental according to whether $u v \bmod \mathfrak{p}$ is a square in $\mathbf{k}^{\times}$or not (here we have used the fact that Char $(\mathbf{k}) \neq 2$.

It is clear that $T^{0}$ leaves the set of characters corresponding to simple strata (resp. split fundamental strata) stable. So we may write

$$
H^{1}\left(\tilde{X}_{2 m+1}\left[s_{0}, c_{0}\right]\right)=H^{1}\left(\tilde{X}_{2 m+1}\left[s_{0}, c_{0}\right]\right)_{\text {simple }} \oplus H^{1}\left(\tilde{X}_{2 m+1}\left[s_{0}, c_{0}\right]\right)_{\text {split }},
$$

where $H^{1}\left(\tilde{X}_{2 m+1}\left[s_{0}, c_{0}\right]\right)_{\text {simple }}$ (resp. $\left.H^{1}\left(\tilde{X}_{2 m+1}\left[s_{0}, c_{0}\right]\right)_{\text {split }}\right)$ is the $T^{0} K_{m}$-submodule which decomposes as a $K_{m} / K_{m+1}$-module as a direct sum of (characters corresponding to) simple non-scalar strata (resp. split fundamental strata).

We have a result similar to Proposition 5.1, whose proof uses the same arguments.

Proposition 6.1. Let $\lambda$ be an irreducible constituent of

$$
\operatorname{Ind}_{T^{0} K_{m}}^{K} H^{1}\left(\tilde{X}_{2 m+1}\left[s_{0}, c_{0}\right]\right)_{\text {simple }} \subset H^{1}\left(\tilde{X}_{2 m+1}\left[s_{0}\right]\right) .
$$

Then the compactly induced representation $c$-ind ${ }_{K}^{G} \lambda$ is irreducible, whence supercuspidal. 
The study of $\operatorname{Ind}_{T^{0} K_{m}}^{K} H^{1}\left(\tilde{X}_{2 m+1}\left[s_{0}, c_{0}\right]\right)_{\text {split }}$ is the aim of the next section.

We are now going to determine the $K$-module structure of $H^{1}\left(\tilde{X}_{1}\left[s_{0}\right]\right)$. Set $\mathbf{G}=\operatorname{PGL}(2, \mathbf{k}) \simeq K / K^{1}$ and write $\mathbf{B}$ and $\mathbf{T}$ for the upper Borel subgroup and diagonal torus of $\mathbf{G}$, respectively. Let $\mathbf{U}$ be the unipotent radical of $\mathbf{B}$. As a $K$-set the set of neighbour vertices of $s_{0}$ is isomorpic to $\mathbb{P}^{1}(\mathbf{k})=\mathbf{G} / \mathbf{B}$.

The graph $\Omega=\tilde{X}_{1}\left[s_{0}\right]$ has for the vertex set the set of paths of the form $\left(s, s_{0}\right)$ or $\left(s_{0}, s\right)$ where $s$ runs over the neighbour vertices of $s_{0}$ in $X$. So the space $C^{0}(\Omega)$ of 0-cochains identifies with the space $\mathcal{F}\left(\mathbb{P}^{1}(\mathbf{k}) \amalg \mathbb{P}^{1}(\mathbf{k})\right)$ of complex-valued functions on the disjoint union $\mathbb{P}^{1}(\mathbf{k}) \coprod \mathbb{P}^{1}(\mathbf{k})$, thus has a $\mathbf{G}$-module $C^{0}(\Omega)$ that is isomorphic to $\mathbf{1}_{\mathbf{G}} \oplus \mathbf{S t}_{\mathbf{G}} \oplus \mathbf{1}_{\mathbf{G}} \oplus \mathbf{S t}_{\mathbf{G}}$, where $\mathbf{1}$ denotes a trivial representation and $\mathbf{S t}$ a Steinberg representation.

The $\mathbf{G}$-set $\Omega^{1}$ is the set of paths of the form $\left(s, s_{0}, t\right)$, where $s$ and $t$ are two different neighbour vertices of $s_{0}$. This $\mathbf{G}$-set is isomorphic to the quotient $\mathbf{G} / \mathbf{T}$. The space $C^{(1)}(\Omega)$ of unoriented 1-cochains identifies as a $G$-module with the space $\mathcal{F}(\mathbf{G} / \mathbf{T})$.

Fix a non-trivial character $\psi$ of $\mathbf{U}$. It is well known that the induced representation $\operatorname{Ind}_{\mathbf{U}}^{\mathbf{G}} \psi$ is multiplicity free. Its irreducible constituent form is by definition the generic (irreducible) representations of $\mathbf{G}$. Moreover, an irreducible representation is generic if and only if it is not a character.

We have a natural $G$-equivariant map $\Phi: \mathcal{F}(\mathbf{G} / \mathbf{T}) \longrightarrow \operatorname{Ind}_{\mathbf{U}}^{\mathbf{G}} \psi$, given by

$$
\Phi(f)(g)=\sum_{u \in \mathbf{U}} f(g u) \bar{\psi}(u), f \in \mathcal{F}(\mathbf{G} / \mathbf{T}), g \in \mathbf{G} .
$$

If a function $f$ lies in the kernel of $\Phi$, then we have $\sum_{u \in \mathbf{U}} f(g u) \theta(u)=0$, for all $g \in \mathbf{G}$ and all non-trivial character $\theta$ of $\mathbf{U}$. Indeed, it suffices to use the fact that the action of $\mathbf{T}$ on $\mathbf{U}$ by conjugation acts transitively on the non-trivial characters of $\mathbf{U}$ and the right invariance of $f$ under the action of $T$. So the kernel of $\Phi$ consists of the function $f$ such that $u \mapsto f(g u)$ is a constant function on $U$, for all $g \in G$. In other words, $\operatorname{Ker} \Phi=\mathcal{F}(G / B) \simeq \mathbf{1}_{\mathbf{G}} \oplus \mathbf{S t}_{\mathbf{G}}$. By a dimension argument, we see that $\Phi$ is surjective. It follows that

$$
C^{(1)}(\Omega) \simeq \operatorname{Ind}_{\mathbf{U}}^{\mathbf{G}} \psi \oplus \mathbf{1}_{\mathbf{G}} \oplus \mathbf{S t}_{\mathbf{G}} .
$$

We have the cochain complex of $G$-modules:

$$
0 \longrightarrow C^{0}(\Omega) \longrightarrow C^{(1)}(\Omega) \longrightarrow 0 .
$$

Since $\Omega$ is connected the kernel of the coboundary operator is the trivial module $\mathbb{C}$. Hence in the Grothendieck groups of $G$-modules, we have $d C^{0}(\Omega) \simeq 2 . \mathbf{1}_{\mathbf{G}}+$ $2 . \mathbf{S t}_{\mathbf{G}}-\mathbf{1}_{\mathbf{G}}=\mathbf{1}_{\mathbf{G}}+2 . \mathbf{S t}_{\mathbf{G}}$. Therefore,

$$
H^{1}(\Omega)=C^{1}(\Omega) / d C^{0}(\Omega) \simeq \operatorname{Ind}_{\mathbf{U}}^{\mathbf{G}} \psi+\mathbf{1}_{\mathbf{G}}+\mathbf{S t}_{\mathbf{G}}-\mathbf{1}_{\mathbf{G}}-2 \cdot \mathbf{S t}_{\mathbf{G}}=\operatorname{Ind}_{\mathbf{U}}^{\mathbf{G}} \psi-\mathbf{S t}_{\mathbf{G}} .
$$

Since $q=|\mathbf{k}|$ is odd, there exists a unique non-trivial character of $\mathbf{k}^{\times} /\left(\mathbf{k}^{\times}\right)^{2}$, that we denote by $\chi_{0}$. The irreducible constituents of the Gelfand-Graev representation $\operatorname{Ind}_{\mathbf{U}}^{\mathbf{G}} \psi$ are the following:

- the irreducible cuspidal representations of $\mathbf{G}$,

- the principal series $\operatorname{Ind}_{\mathbf{B}}^{\mathbf{G}} \chi \otimes \chi^{-1}$, where $\chi: \mathbf{k}^{\times} \longrightarrow \mathbb{C}^{\times}$is a character such that $\chi^{2} \not \equiv 1$ (i.e. $\chi \notin\left\{\mathbf{1}, \chi_{0}\right\}$ ).

- the steinberg representation $\mathbf{S t}_{\mathbf{G}}$,

- (when $q$ is odd) the twisted representation $\mathbf{S t}_{\mathbf{G}} \otimes \chi_{0}$. 
If $\sigma$ is a cuspidal representation of $\mathbf{G}=K / K^{1}$, then the induced representation $c$-ind $K_{K}^{G} \sigma$ is irreducible and supercuspidal ([4, (11.5), page 81]). Such a representation of $G$ is called a level 0 supercuspidal representation.

A principal series of $\mathbf{G}=K / K^{1}$ may be writen as $\operatorname{Ind}_{I}^{K} \rho$, where $\rho$ is a character of $I / I^{1}$. The pair $(I, \rho)$ is actually a type in the sense of Bushnell and Kutzko's type theory. For technical reasons we postpone definitions and references to the next section. Since the representation $\operatorname{Ind}_{I}^{K} \rho$ is irreducible, it is a type of the same constituent as $(I, \rho)$.

To sum up, we have proved the following.

Proposition 6.2. An irreducible constituent $\lambda$ of $H^{1}\left(\tilde{X}_{1}\left[s_{0}\right]\right)$ is of one of the following forms:

(i) the inflation of a cuspidal representation of $\mathbf{G}$; in that case $c$-ind ${ }_{K}^{G} \lambda$ is a level 0 irreducible supercuspidal representation of $G$,

(ii) the inflation to $K$ of the representation $\mathbf{S t}_{\mathbf{G}} \otimes \chi_{0}$,

(iii) a type of the form $\operatorname{Ind}_{I}^{K} \rho$, where the $\rho$ is inflated from a character of $I / I^{1} \simeq$ $\left(\mathbf{k}^{\times} \times \mathbf{k}^{\times}\right) / \mathbf{k}^{\times}$of the form $\chi \otimes \chi^{-1}, \chi^{2} \not \equiv 1$.

Note that in (iii), the pair $\left(K, \operatorname{Ind}_{I}^{K} \rho\right)$ is a principal series type.

\section{The InduCing REPRESENTATIONS - III}

We keep the notation as in the previous section. To determine the structure of $\operatorname{Ind}_{T^{0} K_{m}}^{K} H^{1}\left(\tilde{X}_{2 m+1}\left[s_{0}, c_{0}\right]\right)_{\text {split }}$, we first recall crucial facts on split strata and types for principal series representations. The basic reference for type theory is [6].

Let $\chi$ be a character of $T$, that we view as a character of $T^{0}$ by restriction. Assume that the conductor of $\chi$ is $n>0: T^{n} \subset \operatorname{Ker} \chi$ and $n$ is minimal for this property. Set

$$
J_{\chi}=\left(\begin{array}{cc}
\mathfrak{o}^{\times} & \mathfrak{o} \\
\mathfrak{p}^{n} & \mathfrak{o}^{\times}
\end{array}\right)=\Gamma_{0}\left(\mathfrak{p}^{n}\right)
$$

If $U$ and $\bar{U}$ denote the groups of upper and lower unipotent matrices respectively, then $J_{\chi}$ has an Iwahori decomposition,

$$
J_{\chi}=\left(J_{\chi} \cap \bar{U}\right) \cdot\left(J_{\chi} \cap T\right) \cdot\left(J_{\chi} \cap U\right)
$$

and one may define a character $\rho_{\chi}$ of $J_{\chi}$ by

$$
\rho_{\chi}\left(\bar{u} t^{0} u\right)=\chi\left(t^{0}\right), \bar{u} \in J_{\chi} \cap \bar{U}, u \in J_{\chi} \cap U, t^{0} \in T^{0} .
$$

Let $\mathcal{R}_{[T, \chi]}$ be the Bernstein component of the category of smooth representations of $G$ whose objects are the representations $\mathcal{V}$ satisfying the following property: Any irreducible subquotient of $\mathcal{V}$ occurs in a parabolically induced representation $\operatorname{Ind}_{\mathcal{B}}^{G}\left(\chi \otimes \chi_{0}\right)$, where $\mathcal{B}$ is a Borel subgroup with Levi component $T$ and $\chi_{0}$ an unramified character of $T$. We then have:

Theorem 7.1 (A. Roche). The pair $\left(J_{\chi}, \rho_{\chi}\right)$ is a type for $\mathcal{R}_{[T, \chi]}$.

This is indeed Theorem (7.7) of [11]. Note that our $J_{\chi}$ is not exactly the same as Roche's, but a conjugate under an element of $T$ (see [11, Example (3.5)]).

Proposition 7.2. With the notation as before, assume that $\chi_{\mid T^{0}}$ is not of the form $\alpha \circ$ Det, where $\alpha$ is a character of $\mathfrak{o}^{\times}$(necessarily of order 2). Then the induced representation $\operatorname{Ind}_{J_{\chi}}^{K} \rho_{\chi}$ is irreducible. In particular, it is a type for $\mathcal{R}_{[T, \chi]}$. 
Proof. Let $W$ be the extended affine Weyl group of $G$ w.r.t. $T$ and set $W_{\chi}=\{w \in$ $W ; w \chi=\chi\}$. Then by Theorem (4.14) of [11], the $G$-intertwining of $\rho_{\chi}$ is $J_{\chi} W_{\chi} J_{\chi}$. The hypothesis on $\chi$ forces $W_{\chi}=T / T^{0}$. So $\left(J_{\chi} W_{\chi} J_{\chi}\right) \cap K=J_{\chi} T^{0} J_{\chi}=J_{\chi}$, and we may apply Mackey's criterion of irreducibility.

For $n>0$ and $q \in\{0, \ldots, n\}$, define compact open subgroups of $G$ as follows:

$$
{ }_{q} \mathfrak{h}_{1}=\left(\begin{array}{cc}
1+\mathfrak{p}^{n} & \mathfrak{p}^{q} \\
\mathfrak{p}^{n+1} & 1+\mathfrak{p}^{n}
\end{array}\right) \text { and } \mathfrak{h}_{2}=\left(\begin{array}{cc}
1+\mathfrak{p}^{n+1} & \mathfrak{p}^{q} \\
\mathfrak{p}^{n+1} & 1+\mathfrak{p}^{n+1}
\end{array}\right)
$$

These groups are particular cases of groups considered in 1 , $\S(2.3)]$. The quotients ${ }_{q} \mathfrak{h}_{1} /{ }_{q} \mathfrak{h}_{2}, q=0, \ldots, n$, are abelian, and for $\alpha \in \mathbf{k}^{\times}$, one may define a character $\psi_{\alpha}$ of ${ }_{q} \mathfrak{h}_{1} / \mathfrak{q}_{2}$ by the formula

$$
\psi_{\alpha}\left(I_{2}+\left(\begin{array}{cc}
\varpi^{n} a & \varpi^{q} b \\
\varpi^{n+1} c & \varpi^{n} d
\end{array}\right)\right)=\psi(\alpha(a-d)),
$$

where $\psi$ is a fixed non-trivial character of $(\mathbf{k},+)$. We shall need the following result.

Lemma 7.3. If a smooth representation of $K$ contains $\left(\psi_{\alpha}\right)_{\left.\right|_{n} \mathfrak{h}_{1}}$ by restriction, then it contains the character $\left(\psi_{\alpha}\right)_{\left.\right|_{0} \mathfrak{h}_{1}}$.

Proof. Since the characteristic of $\mathbf{k}$ is not 2 , then $\alpha \neq-\alpha$ and $\left(\psi_{\alpha}\right)_{\left.\right|_{n} \mathfrak{h}_{1}}$ is the restriction to ${ }_{n} \mathfrak{h}_{1}$ of a split fundamental stratum of $K_{n} / K_{n+1}$. Our lemma is then a particular case of [1, Lemma (2.4.5)].

Proposition 7.4. Let $\lambda$ be an irreducible constituent of $\operatorname{Ind}_{T^{0} K_{m}}^{K} H^{1}\left(\tilde{X}_{2 m+1}\right.$ $\left.\left[s_{0}, c_{0}\right]\right)_{\text {split. }}$. Then with the notation as above, $\lambda$ is of the form $\operatorname{Ind}_{J_{\chi}}^{K} \rho_{\chi}$, for some principal series type $\left(J_{\chi}, \rho_{\chi}\right)$ with $\chi$ of conductor $m+1$.

Proof. We know that such a $\lambda$ contains a split fundamental stratum of the form $[\mathrm{M}(2, \mathfrak{o}), m, m-1, b]$, where $b=\varpi^{-m}\left(\begin{array}{cc}0 & u \\ v & 0\end{array}\right), u, v \in \mathfrak{o}^{\times}$, and $u v$ is a square $\operatorname{modulo} \mathfrak{p}$. If $\alpha \in \mathfrak{o}$ is such that $\alpha^{2} \equiv u v \bmod \mathfrak{p}$, then the stratum is equivalent to a $K$-conjugate of $\left[\mathrm{M}(2, \mathfrak{o}), m, m-1, b^{\prime}\right]$, where $b^{\prime}=\varpi^{-m}\left(\begin{array}{cc}\alpha & 0 \\ 0 & -\alpha\end{array}\right)$. So we deduce that $\lambda$ contains this latter stratum by restriction. Now consider the group ${ }_{n} \mathfrak{h}_{1}$ for $n=m$. The representation $\lambda$ contains the character $\left(\psi_{\alpha}\right)_{\left.\right|_{n} \mathfrak{h}_{1}}$ by restriction. By applying Lemma 7.3 we obtain that it contains the character $\left(\psi_{\alpha}\right)_{\left.\right|_{0} \mathfrak{h}_{1}}$. This character clearly extends to $T_{0}^{0} \mathfrak{h}_{1}=\Gamma_{0}(m+1,0)$ and the quotient $T_{0}^{0} \mathfrak{h}_{1} /{ }_{0} \mathfrak{h}_{1}$ is abelian. It follows that $\lambda$ contains an extension of $\psi_{\alpha}$ to $\Gamma_{0}(m+1,0)$. Such an extension is of the form $\left(J_{\chi}, \rho_{\chi}\right)$, for some character $\chi$ of $T$ of conductor $m+1$. The fact that $\lambda$ is induced from $\left(J_{\chi}, \rho_{\chi}\right)$ follows from Proposition 7.2 .

\section{SyNTHESIS}

We now prove Theorems A and B of the Introduction.

By Propositions 4.2 and 4.4, we have isomorphisms of $G$-modules:

$$
\begin{gathered}
H_{c}^{1}\left(\tilde{X}_{2 m}\right) \simeq H_{c}^{1}\left(\tilde{X}_{2 m-1}\right) \oplus c-\operatorname{ind}_{\mathcal{K}_{1}}^{G} H^{1}\left(\Sigma_{2 m}\right), m \geq 1, \\
H_{c}^{1}\left(\tilde{X}_{2 m+1}\right) \simeq H_{c}^{1}\left(\tilde{X}_{2 m}\right) \oplus c-\text { ind }_{\mathcal{K}_{0}}^{G} H^{1}\left(\Sigma_{2 m+1}\right), m \geq 0 .
\end{gathered}
$$

Recall that with the notation of the introduction, we have:

$-\Sigma_{2 m}=\tilde{X}_{2 m}\left[e_{0}\right], \Sigma_{2 m+1}=\tilde{X}_{2 m+1}\left[s_{0}\right]$,

$-\mathcal{K}_{0}=\mathcal{K}_{s_{0}}, \mathcal{K}_{1}=\mathcal{K}_{e_{0}}$. 
Moreover, by Proposition 5.2 we have

$$
H_{c}^{1}\left(\tilde{X}_{0}\right) \simeq \operatorname{St}_{G} \oplus c-\operatorname{ind}_{\mathcal{K}_{1}}^{G} H^{1}\left(\Sigma_{0}\right)
$$

so that (1) holds for $m=0$. Hence, Theorem A follows from (8.1) and (8.2) by a straightforward inductive argument.

Theorem B follows from the discription of the irreducible components of $H^{1}\left(\Sigma_{n}\right)$ given in Proposition 5.1 ( $n$ even and $n>0)$, Proposition $5.2(n=0)$, and Propositions 6.1 and $7.4(n$ odd $)$.

\section{REFERENCES}

[1] Paul Broussous, Minimal strata for GL(m,D), J. Reine Angew. Math. 514 (1999), 199-236, DOI 10.1515/crll.1999.071. MR1711267 (2000m:22020)

[2] Paul Broussous, Simplicial complexes lying equivariantly over the affine building of GL $(N)$, Math. Ann. 329 (2004), no. 3, 495-511, DOI 10.1007/s00208-004-0516-3. MR2127987 (2005m:20117)

[3] Paul Broussous, Representations of PGL(2) of a local field and harmonic cochains on graphs, Ann. Fac. Sci. Toulouse Math. (6) 18 (2009), no. 3, 495-513 (English, with English and French summaries). MR2582439 (2011e:22021)

[4] Colin J. Bushnell and Guy Henniart, The local Langlands conjecture for GL(2), Grundlehren der Mathematischen Wissenschaften [Fundamental Principles of Mathematical Sciences], vol. 335, Springer-Verlag, Berlin, 2006. MR2234120(2007m:22013)

[5] Colin J. Bushnell and Philip C. Kutzko, The admissible dual of GL $(N)$ via compact open subgroups, Annals of Mathematics Studies, vol. 129, Princeton University Press, Princeton, NJ, 1993. MR:1204652 (94h:22007)

[6] Colin J. Bushnell and Philip C. Kutzko, Smooth representations of reductive p-adic groups: structure theory via types, Proc. London Math. Soc. (3) 77 (1998), no. 3, 582-634, DOI 10.1112/S0024611598000574. MR1643417 (2000c:22014)

[7] Armand Borel and Jean-Pierre Serre, Cohomologie à supports compacts des immeubles de Bruhat-Tits; applications à la cohomologie des groupes S-arithmétiques, C. R. Acad. Sci. Paris Sér. A-B 272 (1971), A110-A113 (French). MR0274456 (43 \#221)

[8] H. Carayol, Représentations cuspidales du groupe linéaire, Ann. Sci. École Norm. Sup. (4) 17 (1984), no. 2, 191-225 (French). MR760676 (86f:22019)

[9] G. Henniart, Sur l'unicité des types pour $\mathrm{GL}_{2}$, appendix to Multiplicité modulaires et représentations de $\operatorname{GL}\left(2, \mathbb{Z}_{p}\right)$ et $\operatorname{Gal}\left(\overline{\mathbb{Q}}_{p} / \mathbb{Q}_{p}\right)$ en $l=p$, by C. Breuil and A. Mézard.

[10] P. C. Kutzko, On the supercuspidal representations of $\mathrm{Gl}_{2}$, Amer. J. Math. 100 (1978), no. 1, 43-60. MR0507253 (58 \#22411a)

[11] Alan Roche, Types and Hecke algebras for principal series representations of split reductive p-adic groups, Ann. Sci. École Norm. Sup. (4) 31 (1998), no. 3, 361-413, DOI 10.1016/S00129593(98)80139-0 (English, with English and French summaries). MR1621409 (99d:22028)

Département de Mathématiques, Université de Poitiers, Téléport 2 - BP 30179, Boulevard Marie et Pierre Curie, 86962 Futuroscope Chasseneuil Cedex, France

E-mail address: paul.broussous@math.univ-poitiers.fr 